\author{
Markus D. Meier (Kolumbien)
}

\title{
Reizwort „Darwin“: Eine komparatistische Diskursanalyse zu Wissenschaftskonzepten in zwei gegenwärtigen didaktischen Kontroversen, nebst einem historischen Exkurs
}

\begin{abstract}
Summary (Buzz-word "Darwin": A comparative discourse analysis of concepts of science in two recent didactic controversies, along with a historical excursus): Since schools have left the patronage of religious institutions, the sciences have become their point of reference as far as their didactic content is concerned, the state as far as their form (administration, financiation etc.) is concerned. The present paper aims to show, how political and/or religious interests try to interfere with the scientific orientation in secondary and tertiary education, referring to the case of Charles Darwins theory of evolution. In an introductory analogy, the implementation of "Rassenkunde (race education)" during the Third Reich is reconstructed and contrasted to attempts to install "creationism" resp. "gender-studies" in education today. Both intentions are concludingly evaluated in respect to their scientific and political foundations and implications.
\end{abstract}

Key words: Charles Darwin, creationism, gender-studies, religion and science

Резюме (Маркус Д. Мейер [Колумбия]: Слово-стимул «Дарвин»: сравнительный дискурс-анализ по научным концептам в двух современных дидактических дискуссиях, наряду с историческим экскурсом): С тех пор как институты образования все больще и больше выделялись из религиозных учреждений, то науки в содержательно-дидактическом отношении, а государство в формальном (административном, финансовом и т.д.) отношении становились центральными пунктами образования. Статья на примере эволюционной теории Чарьза Дарвина показывает, как политические и/или религиозные интересы и сегодня пытаются оказать влияние на научню ориентацию вторичного и третичного образования. По исторической аналогии производится реконструкция введения «Расового учения» в Третьем Рейхе и сравнение с попытками реализации «креационизма» и/или «гендерного учения» в современном образовании. В заключение обе попытки оцениваются в отношении политических и научных основ и импликаций.

Клучевые слова: Charles Darwin, Креационизм, гендерные исследования, религия и наука

Zusammenfassung: Seitdem Bildungsinstitutionen zunehmend aus religiösen Institutionen herausgelöst wurden, sind die Wissenschaften in inhaltich-didaktischer und der Staat in formaler (administrativer, finanzieller etc.) Hinsicht zu zentralen Referenzpunkten von Bildung geworden. Der Artikel möchte am Beispiel von Charles Darwins Evolutionstheorie zeigen, wie politische und/oder religiöse Interessen auch heute noch auf die Wissenschaftsorientierung von sekundärer und tertiärer Bildung Einfluss zu nehmen versuchen. In einer einführenden historischen Analogie wird die Einführung von "Rassenkunde" im Dritten Reich rekonstruiert und mit Versuchen, "Kreationismus" bzw. "Genderstudien" in gegenwärtiger Bildung zu implementieren, konstrastiert. Beide Versuche werden abschließend im Hinblick auf ihre politischen und wissenschaftlichen Grundlagen und Implikationen bewertet.

Schlüsselwörter: Charles Darwin, Kreationismus, Genderstudien, Religion und Wissenschaft 


\section{Einleitung}

Seit ihrer organisatorischen und curricularen Herauslösung aus ekklesialen Kontexten hat (zunehmend staatlich finanzierte) schulische Bildung auch eine Ablösung von theologischmetaphysischen Inhalten angestrebt, die durch wissenschaftliche Paradigmen ersetzt werden sollten und wurden. Verschiedene Kriterien wurden und werden dabei an Wissenschaftlichkeit gestellt: Ihr Gegenstand muss klar definierbar und definiert sein, der theoretische Rahmen muss begründbar sein und begründet dargestellt werden, Ergebnisse müssen empirisch falsifizierbar sein um evtl. eine Neuformulierung wissenschaftlicher Erkenntnisse und Bildungsinhalte zu ermöglichen in einer Fachsprache, deren Kategorien sauber trennbar, logisch nachvollziehbar, widerspruchsfrei und sprachlich - nicht unbedingt spontan, aber prinzipiell - klar und verständlich sind (ausführlich Lauth \& Sareiter, 2002; zum hier zugrunde liegenden Wissenschaftsverständnis Popper, 1935). Ausgeschlossen sind wissenschaftstheoretisch Rekurse auf Autoritäten und ihre "Heiligen Texte", aber auch solche auf die faktische Definitionsmacht von edukativen religiösen oder politischen Institutionen (Kirchen oder Ministerien etwa). Die Fachdidaktiken der Schulfächer müssen sich durch diese Wissenschaftsorientierung immer wieder neu orientieren, damit "Schulwissen" nicht von der fortschreitenden wissenschaftlichen Entwicklung der jeweiligen Disziplinen abgekoppelt wird. Tun sie dieses nämlich nicht, so laufen sie Gefahr religiös und/oder politisch erwünschte Dogmen in Unterrichtsinhalte zu verwandeln, die den Kriterien ihrer jeweiligen wissenschaftlichen Diskurse nicht entsprechen bzw. nicht mehr entsprechen.

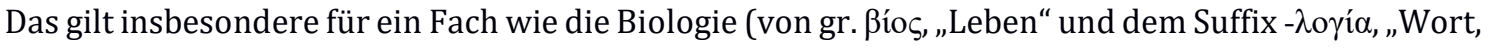
Lehre“), die als „Lebens-Kunde“ (ihrer Definition nach) viele Anknüpfungspunkte an Referenzdiskurse bietet. So ist die "Frage nach dem Leben" eben zentral nicht nur in der Theologie und Philosophie, sondern auch in den Sozialwissenschaften - und der Politik. Es nimmt daher nicht Wunder, dass von diesen beiden Seiten - also Strömungen innerhalb der Theologie einerseits und innerhalb der Sozialwissenschaften, aber auch der Bildungspolitik andererseits - gegenwärtig massiv Einfluss auf die Ausgestaltung nicht nur biologischer Didaktik genommen werden soll und teilweise genommen wird.

Methodisch versteht sich der Beitrag als Übung in dem, was Berger und Luckmann in Anlehnung an Nietzsche „die Kunst des Misstrauens“ (Berger \& Luckmann, 1968/2003, S. 7) genannt haben. Wir wollen nachweisen, dass der pure Verweis auf die triviale Tatsache, dass Wissensproduktion als gesellschaftlicher - in unserem Fall erziehungswissenschaftlicher - Prozess zu verstehen sei (dass also Publikationen nicht etwa „vom Himmel fallen“, oder einfach „erscheinen“ ...), in seinem bescheidenen epistemologischen Anspruch selbst zu Ideologie und Ressentiment dann wird, wenn er sich jeglichen Bezugs zu theoriegelenkter Betrachtung empirischer Realität enthält - zu nicht nur unwissenschaftlichem, sondern unsinnigem „anything goes“. Der Verweis resultiert dann in wenig erkenntnisversprechenden, oft plumpen Versuchen, das filigrane Gefüge von historischer und systematischer Dialektik, das erst die fragile und faszinierende Vielfalt wissenschaftlicher Erkenntnis „intern“ ermöglicht, durch Verweise auf ihre Einbettung in v.a. staatliche Machtstrukturen „extern“ zu erschüttern, zu relativieren, evtl. zu zerstören - den immer wieder neu geknüpften und gelösten gordischen Knoten der Wissenschaft mit dem imposanten Schwert politischer Einflussnahme zu zerschlagen. In einer Diskursanalyse seien in diesem Sinne zwei aktuelle und ein historisches Beispiel machtgestützter und -geschützter akademischer Debatten de-konstruiert und ihrerseits auf ihre jeweiligen religiösen, gesellschaftlichen, politischen und nicht zuletzt akademischen Herrschaftsabsichten befragt. 
Die für Diskursanalysen zentrale Schwierigkeit der Auswahl eines repräsentativen Untersuchungsmaterials (vgl. Jäger 2011) haben wir folgendermassen gelöst: Günthers Text ist in den 1920/30er Jahren vielfach wiederaufgelegt worden, seine "Bedeutung" zeigt sich nicht zuletzt an der Hertz'schen Gegenschrift; er war "der Rassepapst" der Nationalsozialisten. Die Positionen des Kreationismus fristen in der Wissenschaft kaum ein Schattendasein, eine Paraphrase und der Verweis auf die Argumentation der Kultusministerin erschien uns deshalb ausreichend, um sie nachvollziehbar zu rekonstruieren. Am schwierigsten fiel die Auswahl im dritten Fall: Der SielerText arbeitet paradigmatisch an der Schnittstelle von Gender- und Queerstudien und profitiert dabei in seiner Argumentation von beiden Richtungen, ausserdem macht auch hier das Publikationsmedium die enge Verzahnung des Diskurses mit (Bildungs-)politik deutlich, der für unsere Argumentation zentral ist; das gleiche gilt für den anonymen Text des NRWMinisteriums. Gerade beider paraphrasierender Charakter und ihr Fokus auf bildungspolitischen Fragen als "Kern" ermöglichen es, sich nicht in der Analyse "scholastischer" argumentativer Artefakte zu verlieren, die ansonsten diesen hochinstitutionalisiert-selbstreferentiellen Diskurs prägen.

Versuche einer Einflussnahme auf Wissenschaft „Von außen“ sind nicht neu. Einen beunruhigenden historischen Vorläufer haben sie z.B. in der Biologischen Rassekunde im Nationalsozialismus, der die Biologie zu einem Kernfach nationalsozialistischer Bildungspolitik erhob (Wegner, 1991) und theoretisch unbegründete, definitorisch unklar-unsinnige, empirisch nicht fundierte und insofern unwiderlegbare rassistische Glaubenssätze mit ihrem je eigenen sprachlichen Duktus im schulischen und universitären Kontext implementierte - mehr noch: Gerade diese ihre unwissenschaftlichen Charakteristika, ihre logisch unzugängliche und sprachlich nebulöse, ihre sozusagen unbestimmtunmittelbare Unsinnigkeit, sollte ihren nur intuitiv, nicht rational erfassbaren Wert verbürgen. Ein historischer Exkurs stehe am Anfang der Untersuchung.

Karriere und Werk des „Rassentheoretikers“ Hans Friedrich Karl Günther, eines promovierten Germanisten und Studienrats ohne biologische, insbesondere evolutionsbiologische Fachkenntnisse, der sich als politischer Schriftsteller empfand, stehen beispielhaft für eine Entwissenschaftlichung und Politisierung der deutschen Hochschulen und Schulen in diesen 30'er Jahren des vergangenen Jahrhunderts. Im Sinne eines hear-one-hear'em-all ein Textbeispiel:

\begin{abstract}
„Wenn Deutschtum nicht so viel bedeutet wie: Streben zu nordischem Geist, dann bleibt nur eine Verneinung übrig: Abfall von nordischem Geist. Ein neues Streben aber, etwa ein Streben zu ostischem Geist, ist nicht möglich, da es so etwas wie ostischen Geist nicht gibt oder da ostischer Geist eben durch alle Umzüchtung kaum noch mehr bedeuten kann als: Anpassung an die Führung artfremden Geistes, Einordnung in Gemeinschaftsgebilde, welche von artfremden Menschen, von nordischen Menschen oder - wie in den abendländischen Grossstädten - von vorwiegend vorderasiatischen und vorwiegend nordischen Menschen geschaffen sind." (Günther 1935, S. 463)
\end{abstract}

Die Terminologie bezüglich des „Strebens und Abfalls von nordischem Geist“ ist unklar, der Argumentationsgang bezüglich des „nicht existenten und aber umgezüchteten ostischen Geistes" widersprüchlich, die Schlussfolgerungen unklar-beliebig; lediglich die politische Absicht „hinter“ dem krypto-biologischen Text ist unmittelbar und aufdringlich zugänglich. 1930 wurde Günther von einer halben Stelle im sächsischen Schuldienst auf einen neugeschaffenen „Lehrstuhl für Sozialanthropologie“ in Jena berufen - gegen den expliziten Protest vieler deutscher Ordinarien - durch den nationalsozialistischen thüringischen Ministerpräsidenten Frick, die Antrittsvorlesung besuchten ostentativ Adolf Hitler und Hermann Göring. 
Noch im selben Jahr verfasste der österreichische Nationalökonom Friedrich Hertz eine Protestschrift „Hans Günther als Rassenforscher“ (Hertz, 1930) und wurde von seinen ideologischen Gegnern umgehend - und durchaus zutreffenderweise - als „Jude, Freimaurer und Pazifist" diffamiert. Hertz selbst verstand sich als soziologisch argumentierender Minderheitenforscher, der auf die Kraft des besseren Arguments gerade auch gegenüber politischer Erpressung setzte, nach dem Krieg engagierte er sich folgerichtig stark für die entrechteten und vertriebenen "Sudetendeutschen" aus Böhmen und Mähren. Seine wissenschaftliche Argumentation hatte (hochschul-)politischer Agitation jedoch letztlich nichts entgegenzusetzen, wie Günthers erfolgreiche Berufung und Hertz' eigene Emigration 1938 zeigt (alle Angaben nach Schwandt, 2008).

Dieser kurze historische Exkurs zum Thema möge beispielhaft beleuchten, welche verheerenden Auswirkungen eine fachfremde, ideologisch motivierte Politisierung und Entwissenschaftlichung von Hochschulen zeitigen kann. Sie zerstört die Grundparadigmen wissenschaftlichen Arbeitens, nämlich die Überzeugung von der personenindifferenten, methodisch gelenkten empirischen Falsifizierbarkeit von Aussagen und ersetzt sie durch den impliziten oder expliziten, in jedem Fall jedoch wissenschaftlich gesehen irrelevant-unredlichen Verweis auf den jeweils machtpolitisch stärkeren. Ein Versagen der Hochschulen und Schulen in dieser Zeit bestand darin, nicht ausreichend zu reflektieren, dass Wissensproduktion in politische Systeme eingebunden ist und es daher unverzichtbar gewesen wäre, dieser Aufkündigung wissenschaftlicher Argumentationsgrundlagen entschieden entgegenzutreten - und sie nicht zeittypisch „unpolitisch“-achselzuckend abzutun - , da auf eine Habermas'sche „Eigen-Einsicht in das schwächere Argument“ nicht vertraut werden konnte; Günther veröffentlichte noch nach Kriegsende ohne Pseudonym Warnungen vor Ehen mit Zuckerkranken, Frauenrechtlerinnen und Gewohnheitstrinkern (Schwandt, 2008).

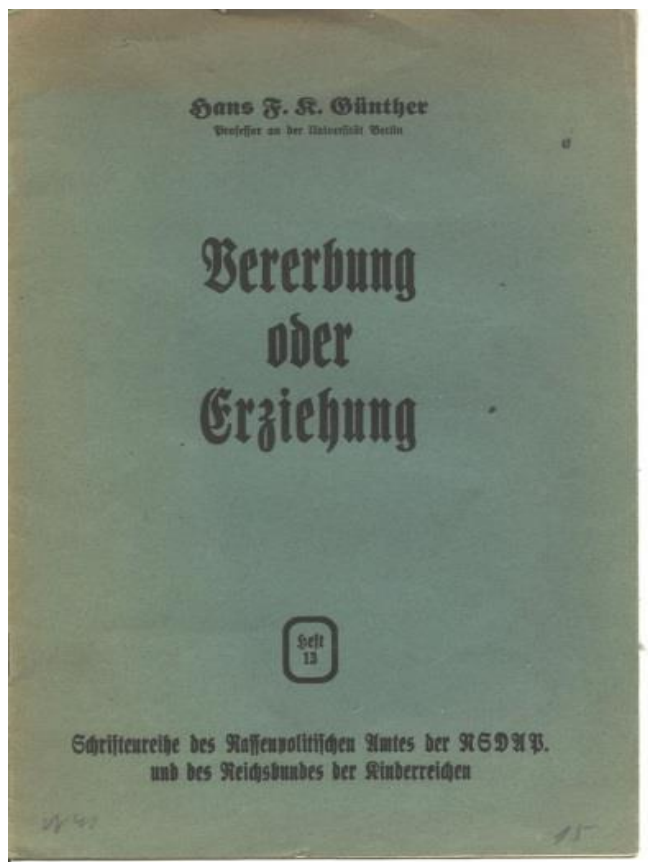

Abbildung 1: Hans Friedrich Karl Günther, Vererbung oder Erziehung

$\mathrm{Zu}$ einem Kristallisationspunkt der Auseinandersetzung um Biologie zwischen wissenschaftlichen Standards und religiöser Indienstnahme hat sich in den letzten Jahren die Debatte um den sogenannten „Kreationismus“ entwickelt. Diese Debatte findet im Spannungsfeld eines wörtlich verstandenen, „verbalinspirierten“ Tora-, Bibel-, oder Koranglaubens und der modernen, universitär 
verankerten Evolutionstheorie im Gefolge v.a. Charles Darwins statt. Beschränken wir uns in der Darstellung auf die politisch einflussreichen fundamentalistisch-kreationistischen Christen.

Die beiden alttestamentarischen Schöpfungserzählungen der Genesis, v.a. aber die „Wortgezeugtheit der Welt", wie sie das Johannesevangelium beschreibt, werden als der Vorstellung von der „Entstehung der Arten durch natürliche und sexuelle Selektion“ diametral entgegengesetzt verstanden. Da die Bibel - als „Heilige Schrift" - definitionsgemäss und in jedem Fall nicht falsifizierbar sei, sondern wörtlich zu verstehendes Wort Gottes, wird sie als „Widerspruch“ zur wissenschaftlichen Evolutionslehre gelesen, oder anders herum ausgedrückt: Der Evolutionslehre und ihren „Anhängern“ wird vorgeworfen, sie lehnten den christlichen bzw. jüdischen Glauben ab, sie seien letztlich Herätiker. Dieser Konflikt lässt sich naturwissenschaftlich-argumentativ nicht lösen, sondern nur systemtheoretisch im Sinne einer "dekretierten Anschlussunfähigkeit" verstehen. Als eine solche „alternative Sichtweise“ soll Kreationismus denn auch unkommentiert statt (bzw. neben) der Evolutionslehre in der Biologie v.a. schulpolitisch-fachdidaktisch verankert und gelehrt werden. (Nota bene: Diese schulpolitische Forderung ist v.a. eine US-Konstellation, denn in den USA herrscht durch die Trennung von Kirche und Staat ein Verbot von Religionsunterricht an Schulen, in die kreationistisches Gedankengut - wenn überhaupt - letztlich besser „passen“ würde.)

Gegen diese Implementierung kreationistischen Gedankengutes im Rahmen wissenschaftsorientierter Biologie aber gibt es gewichtige wissenschaftliche und wissenschaftstheoretische Einwände, die kurz wiederholt seien.

Die Vorstellung von der Erschaffung der Welt in 7 Tagen widerspricht - „verbalinspiriert“-wörtlich genommen - jeglicher archäologischer, geologischer, biologischer und anthropologischer Einsicht. Versteht man „7 Tage“ hingegen metaphorisch im Sinne einer „Prozessualisierung der Menschwerdung“, so ist sie durchaus mit Darwins Evolutionslehre nicht nur vereinbar. Sie kann dann - aber auch nur dann - geradezu als eine uralte, von dem der empirischen Erfahrung zugänglichen Wissen ihrer Zeit ausgehende, frühe Evolutionslehre gelten. Ein interessanter historischer Text sozusagen, der „historisch-kritisch und entmythologisiert“ Einblick gibt in die Organisation von Wissen in einer längst vergangenen Zeit. Auch die Erzählung von Adam und Eva ergibt so - aber eben wiederum auch nur so - einen epistemologischen Sinn. Die (Selbst-) erkenntnis des Menschen geht von der Erkenntnis seiner eigenen, biologischen Zweigeschlechtlichkeit unmittelbar aus, in ihr erkennt er und sie ihre Sterblichkeit als jeweils kurzfristige Nur-Mann und Nur-Frau, abgetrennt vom unbestimmt-unmittelbaren Sein (Gottes). Nicht biologisch-körperlich, sondern epistemologisch„geistig“ werden Adam und Eva aus dem Paradies unbewussten So-seins in die Wüste der je eigenen, „nackten“ Existenz vertrieben, ein Zurück in einen Bereich unbewusster Selbstvergessenheit gibt es für Menschen seitdem nicht (mehr). Adam und Eva sind Allegorien für die conditio humana, die Notwendigkeit, aus der räumlich und zeitlich begrenzten, geschlechtlich dimorphen, immer körperlich-verletztlichen Existenz „im Schweiße (ihres) Angesichts“ etwas zu machen (1. Mose 3:19, vgl. ausführlich Meier 2015, S. 19-23). Und auch die Paraphrasierung stoischer Philosophie zu Beginn des Johannesevangeliums, die Vorstellung einer „Wort“-gezeugtheit der Welt „am Anfang“ - eines „Logos“-Masterplans für die Erschaffung der Welt und alles Lebens sozusagen (eine Kernvorstellung des Kreationismus) - ist wissenschaftlich nicht falsifizierbar, denn was „vor“ dem Urknall war, ob er sozusagen „göttlich geplant“, oder „naturwissenschaftlich passiert“ ist, zu dieser meta-physischen Frage nach „Raum und Zeit vor dem Entstehen/der Erschaffung von Raum und Zeit“ kann sich moderne Biologie nicht äußern, da sie empirisch (bisher) nicht zugänglich ist. Das gilt im Übrigen bekanntlich für alle biologischen „Wozu“-Fragen, die teleologisch-final ausgerichtet sind, Mephistopheles' Eingangsfrage „Wozu der Lärm?“ ist heute so unbeantwortet wie zu Goethes - oder jeder anderen - Zeit. 


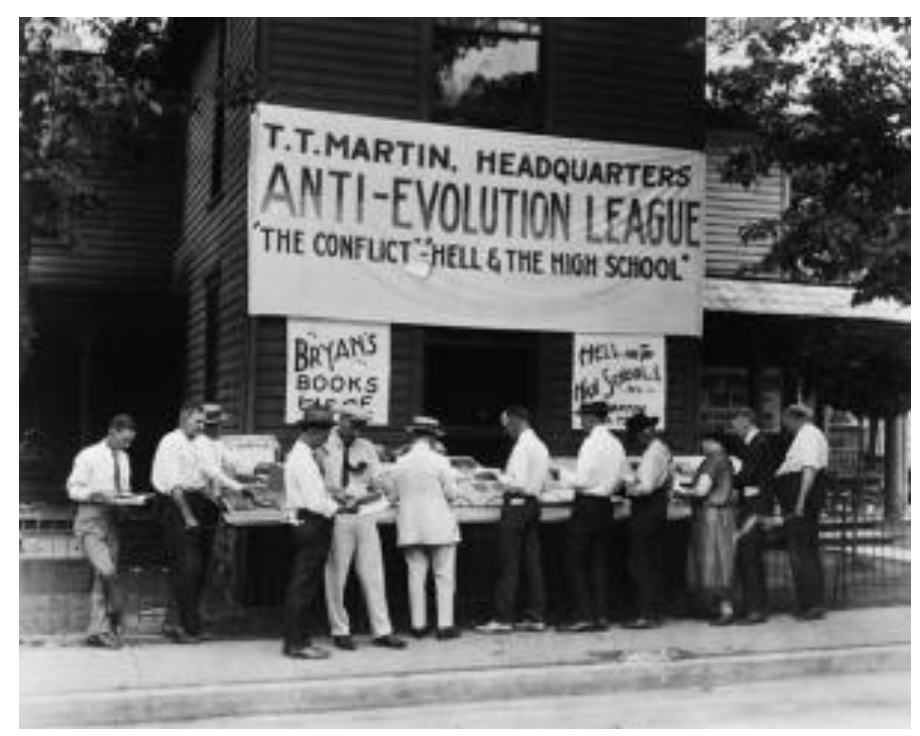

Abbildung 2: Stand einer „Anti-Evolutions-Liga“ in den Südstaaten der USA, 1925

Positiv ist in der Kreationismusdebatte hervorzuheben, dass es einen überwältigenden bildungspolitischen Widerstand v.a. in Europa, aber auch den Vereinigten Staaten gibt. Die Implemetierung kreationistischer Lehre, einer wörtlich verstandenen Auslegung biblischer Texte mitsamt eines Postulats ihrer Überlegenheit gegenüber modern-wissenschaftlicher Erkenntnis, wird zurückgewiesen und als Versuch gewertet, eine - auch theologische (vgl. etwa EKD, 2008) Minderheitenansicht konservativer v.a. evangelikaler Christen, orthodoxer Juden und Muslime politisch „durchzusetzen“ und dadurch die Wissenschaftsorientierung von Schulen zu relativieren. Höchstrichterliche US-Rechtsprechung hat bisher immer gegen die Berücksichtigung von Kreationismus im Biologieunterricht geurteilt, ja - fast will es scheinen, als biete die Kreationismusdebatte philosophierenden und politisierenden Wissenschaftlern eine willkommene Plattform fachfremd ihrerseits dogmatisch-atheistische Positionen zu illustrieren, ja sich in GalileoManier günstig und etwas aufdringlich „in Pose zu werfen“ (vgl. etwa Dawkins, 2006, 2010). Auch in Deutschland sind bildungspolitischen Vorschlägen, die auch nur entfernt an Kreationismus erinnern, keine Erfolge beschieden, selbst wenn sie wissenschaftstheoretisch-explizit lediglich vorschlagen, im Rahmen von Interdisziplinarität die Grenze der diskursiven Systeme Theologie und Biologie selbst zum Inhalt zu machen. So die damalige hessische Kulturministerin Karin Wolff 2007 im Rahmen einer nur sehr kurzen schulpolitischen Debatte, die die Gegenüberstellung von Schöpfungsmythos und Evolutionslehre im Biologieunterricht der Oberstufe - explizit getrennt - vorsah:

„Die Kultusministerin berief sich darauf, dass das Hessische Schulgesetz ein fächerübergreifendes Lernen vorsieht. Schülerinnen und Schülern müsse die Möglichkeit gegeben werden, zwischen der naturwissenschaftlichen und der theologischen Erklärung der Welt zu vergleichen. „Naturwissenschaft erklärt, wie die Welt entstand, bei der Theologie geht es um Deutungs- und Sinnfragen“, so Wolff." (WELT vom 5.7.2007)

Der bildungspolitische Konsens und die Vehemenz, mit der unwissenschaftlich-dogmatisches Denken im Falle des Kreationismus-Streites in Deutschland aus dem schulpolitischen Diskurs gedrängt wurde, machten deutlich, dass es zu einer Wissenschaftsorientierung im Falle konkurrierender fundamentalistisch-religiöser Ansichten keine Alternativen geben solle. Eine fachwissenschaftliche Diskussion gab es zu diesem Thema aus eben diesem Grunde von vorneherein ohnehin nicht. Der Versuch, wie auch immer argumentativ verpackt, religiös begründete Themen in 
naturwissenschaftliche Kontexte einzuführen war gescheitert, Wolff trat kurze Zeit später (auch aus anderen Gründen) zurück.

Dieser Konsens ist im Falle einer ähnlich gelagerten diskursiven Unvereinbarkeit von Biologie und in diesem Fall - politisch-weltanschaulichem Schrifttum wesentlich geringer ausgeprägt. Die Rede ist von der Genderisierungsabsicht nicht nur, aber auch des Biologieunterrichts, v.a. des Sexualkundeunterrichts. Epistemologisch geht dieses gender-Schrifttum von den wissenschafts- und modernitätskritischen Arbeiten der französischen Poststrukturalisten (v.a. Foucaults) aus, radikalisiert diese aber zu einem unwissenschaftlichen "anything goes“ in dem Sinne, dass theoretischen Annahmen (seien sie naturwissenschaftlicher oder geisteswissenschaftlicher Art) kein Falsifizierungspotential durch empirische Belege mehr gegenübergestellt werden könne. Jedes Sprechen - insbesondere auch wissenschaftliches - sei ein interessegeleitetes Erkenntnis-Konstrukt in einer ansonsten unerkennbaren Welt. Erkenntnis könne nur in einer - letztlich solipsistischen De-konstruktion dieser Konstruktion(-en) bestehen, die sich in einem regressum ad infinitum immer weiter fortsetzen lasse. Einen Unterschied zwischen Alltagssprechen, Ideologie und Wissenschaft verneint diese erkenntnistheoretische Richtung. Insbesondere im Bereich der Geschlechterforschung hat diese „Wissenschaft ohne wissenschaftlichen Anspruch“ unter dem Namen gender-studies, gender-mainstreaming, queer-studies, Frauenforschung, Frauen-und Geschlechterforschung, Intersektionsforschung etc. einen erheblichen politischen und inzwischen akademischen Einfluss gewonnen, ihre „Institutionalisierungserfolge“ (Ute Kaiser) sind unübersehbar. Dieses ist sowohl wissenschaftstheoretisch, sowie biologisch-fachwissenschaftlich, aber auch bildungspolitisch und gesamtgesellschaftlich erstaunlich.

Zum einen hat einen pur „rationalistischen“ Konstruktivismus bereits Kant in seiner Kritischen Philosophie ausgeschlossen: Ein epistemologisches Modell, welches sich Begriffe bediene, die sich jeglichen Rückgriffs auf die Empirie entschlage, sei „leer“ - Dekonstruktivismus insofern lediglich ein perpetuum mobile sinnloser Begriffszertrümmerung abseits von Einsicht, eine „Kampfvokabel“ ohne Erkenntniswert (vgl. etwa Hacking, 1999).

Ohne Sinnlichkeit würde uns kein Gegenstand gegeben und ohne Verstand keiner gedacht werden. Gedanken ohne Inhalt sind leer, Anschauungen ohne Begriffe sind blind. Daher ist es eben so nothwendig, seine Begriffe sinnlich zu machen (d. i. ihnen den Gegenstand in der Anschauung beizufügen), als seine Anschauungen sich verständlich zu machen (d. i. sie unter Begriffe zu bringen). Beide Vermögen oder Fähigkeiten können auch ihre Functionen nicht vertauschen. Der Verstand vermag nichts anzuschauen und die Sinne nichts zu denken. Nur daraus, daß sie sich vereinigen, kann Erkenntniß entspringen. (Kant 1781/1976, S. 75)

Biologisch-fachwissenschaftlich hat die Evolutionstheorie in den letzten Jahrzehnten eine erstaunliche Erweiterung in den Bereich menschlichen Verhaltens insgesamt, in den Bereich menschlichen Sexualverhaltens im Besonderen erfahren, die Charles Darwins Evolutionstheorie ausgebaut und gefestigt haben. Diese Erkenntnisse gehen davon aus, dass die Trennung der höheren Organismen in männliche und weibliche mit ihrem verlängerten Reproduktionszyklus (gegenüber Parasiten, Viren, Bakterien etc.) zusammenhängen (Voland, 20093). Männlich und weiblich sind ethisch gesehen keine moralischen Kategorien und epistemologisch gesehen keine sozialen Erfindungen, sondern die schiere Voraussetzung für die Existenz von Menschen, sowohl genotyp (als homo sapiens) wie phänotyp (als Sohn/Tochter von). Es ist von einem biologischfachwissenschaftlichen Standpunkt aus unsinnig, von der Existenz von keinem oder unendlich vielen Geschlechtern auszugehen, wie es die Genderstudien vorschlagen (s.u.). Es widerspricht nicht nur jeglicher Alltagserfahrung - auch die Kugelgestalt der Erde widerspricht Alltagserfahrung ohne deshalb falsch zu sein -, sondern jeglichem biologischem Grundwissen auch heute noch. Denn 
Zweigeschlechtlichkeit wird kulturell gestaltet, sie führt zu sozialen Systemen und ihrer sprachlichen Reflektion, ein großer Teil der Weltliteratur dreht sich geradezu um dieses Thema - Paris stürzt Troja in den Untergang wegen Helenas Schönheit, Äneas verlässt Dido um Rom zu gründen, Kriemhild rächt Siegfried, Faust schwängert Gretchen und Anna Karenina wirft sich vor den Zug ... keineswegs jedoch führt Kultur zu biologischer Zwei-, Vielfach-, Un- oder sonst irgendeiner Geschlechtlichkeit (so etwa Butler 1991, S. 149).

Vielmehr ist Genderismus und die Vorstellung einer sozialen Konstruiertheit von Geschlecht, die dem biologischen Geschlecht „Vorausginge“, genau wie Rassentheorie und Kreationismus, ein Glaubenssatz, der nicht widerlegt werden kann und soll, sondern implementiert. In scheinbar freundlich-onkelhaftem Ton etwa formuliert das nordrhein-westfälische Schulministerium in dieser Sache bewusst nebulös:

Das Besondere [sic!] an der neuen Ausrichtung der Gleichstellungspolitik ist, dass sie nicht von sichtbaren oder vermuteten Defiziten ausgeht und entsprechend situations-bezogen Abhilfe schafft. Die Gender-Strategie setzt vielmehr viel früher und als generelles Leitkriterium an: schon bei der Planung und Entwicklung, aber auch bei der Durchführung und Evaluation einer Maßnahme wird untersucht, ob und in welcher Weise sie Frauen und Männer - unmittelbar oder mittelbar - betrifft. [Zwischenfrage: Wen sollten genderpolitische Maßnahmen denn unmittelbar oder mittelbar betreffen, wenn nicht Männer und Frauen?] Ungleichheiten werden mit der Fragestellung überprüft, ob dadurch Nachteile für die eine oder andere Zielgruppe - unmittelbar oder mittelbar - entstehen und welche Lösungsalternativen zu entwickeln sind. Je nach Ergebnis kann von vornherein eine politische Entscheidung, ein Gesetz oder eine Umsetzungsmaßnahme mit größerer Treffsicherheit auf die Bedürfnisse einer Zielgruppe ausgerichtet werden. Aufwändige Nachbesserungen bei Fehlentwicklungen werden damit vermieden. (Ministerium für Schule und Weiterbildung des Landes Nordrhein-Westfalen, 2015)

Der explizite Verzicht auf die konkrete Definition von sichtbaren oder auch nur vermuteten, evtl. vermutbaren Defiziten, ja auf konkret-,,situationsbezogene Abhilfe(bedürfnisse)“ überhaupt, der hier fröhlich-schillernd als „das Besondere“ apostrophiert wird, macht die „Gender-Strategie“ zu einem unwiderlegbaren Glaubenssatz für beliebige pädagogische Maßnahmen, indem sie sich selbst pauschal-universell sowohl Handlungsbedarf, wie auch Handlungskompetenz und -legitimiät bei „Planung und Entwicklung, (...) Durchführung und Evaluation von Maßnahmen“ bescheinigt, solange Männer und Frauen mittelbar oder unmittelbar betroffen sein könnten. Gender gleicht, gerade in seiner orakelhaft-unbestimmten (erziehungs-)wissenschaftsaffinen Terminologie, einem bildungspolitischen Blankoscheck - und so ist es denn wohl auch gemeint. Was aber „Fehlentwicklungen“ sind, entscheidet der Souverän und nicht die Politik! Das gilt umso mehr, als diese Art Glaubenssätze von politischer und schulpolitischer Seite in der Tat in den Schulen ohne parlamentarische Debatte, aber auch - und das ist hier entscheidend - vollständig konträr zu jeglichem biologischen Alltags- und Fachwissen in Lehrplänen u.a. des Biologieunterrichts verankert werden sollen. Die Bundeszentrale für gesundheitliche Aufklärung etwa fordert in diesem Zusammenhang entsprechend:

„Die kritische [sic!] Anwendung der Gender-Debatte auf Sexualpädagogik und Sexualaufklärung kommt nicht umhin, die in der feministischen Geschlechterforschung herausgearbeitete heterosexuelle Matrix der Dreieinigkeit von Sex, Gender und Begehren als wesentliche Stütze der Zweigeschlechtlichkeit (...) zu thematisieren. Wenn nämlich der Beitrag des Konstruktivismus bzw. Dekonstruktivismus zur Geschlechterforschung ernst genommen und akzeptiert wird, dass „wir nicht nur als Frauen (und gegebenenfalls als 
Männer) diskriminiert und benachteiligt werden, sondern auch dadurch, daß wir Frauen oder Männer zu sein haben“ (...), müssen auch alle damit zusammenhängenden Verhaltensmuster und Erwartungen in Frage gestellt werden. Das heißt also auch, Heterosexualität, Generativität und Kernfamilie zu „entnaturalisieren“ und Sexualpädagogik daraufhin zu überprüfen, inwiefern sie die Möglichkeit zur selbstbestimmten Lebensführung einschränkt, wenn durch ihre Intentionen und Maßnahmen explizit oder implizit nahe gelegt wird, heterosexuell und in Kernfamilien mit leiblichen Kindern zu leben." (http://forum.sexualaufklaerung.de/index.php?docid=667, Aufruf vom 25.9.2015)

Der Autor Uwe Sielert ist Professor für Erziehungswissenschaft an der Universität Kiel u. a. mit Schwerpunkt Sexualpädagogik, Mitglied der Kommission zur „Sexualethik der Evangelischen Kirche“ und der Kommission „Sexualität, Gewalt und Pädagogik“ der DgfE. Er hat es über Genderthemen zu Macht und Einfluss innerhalb der universitären Erziehungswissenschaft und darüber hinaus in der (Bildungs-)politik gebracht (vgl. Schmelcher, 2014). Sielert arbeitet in seiner Argumentation mit unklaren Begriffen: Belege für, oder auch nur eine Explikation des Konstruktes einer „heterosexuelle(n) Matrix der Dreieinigkeit von Sex, Gender und Begehren als wesentliche Stütze der Zweigeschlechtlichkeit" werden nicht vorgestellt, es bleibt beim derogativen Ton biologisch-fachwissenschaftlich ist seine Argumentation unsinnig (s.u.). Die Implementierung einer „entnaturalisierenden“ Sexualerziehung propagiert pragmatisch-faktisch die Disposition der Familienbindung $\mathrm{zu}$ Gunsten von Homosexualität und Pädophilie (der Begriff der „Generativität“ schillert eigentümlich zwischen „Generationalität“ und „Reproduktivität“ und so doppelsinnig ist er wohl auch gemeint), legitimiert diese als Entgrenzung hin zur „Möglichkeit zur selbstbestimmten Lebensführung“, explizit und lege artis abgesichert durch den Verweis auf die durch akademische „feministische Geschlechterforschung herausgearbeitete“ Forschungslage, um die man „nicht umhin(komme)“. Die Annahme aber, Genderismus und die Annahme der sozialen Konstruiertheit von Geschlecht sei „wissenschaftlich“ abgesichert, gar ein Orientierung bietendes aber auch erheischendes wissenschaftliches Programm, ist irrig. Genderismus ist v.a. ein (sexual-) politisches Programm, das sich durch Institutionalisierungserfolge an den Schnittstellen von Bildungspolitik und Erziehungswissenschaft mit erheblichem wissenschaftsimitierend-rhetorischen Aufwand und ohne ausreichende empirische Absicherung etabliert hat.

Die Grundannahme des Genderismus, Geschlecht sei zuallererst eine soziale Kategorie und Zweigeschlechtlichkeit eine historisch-fragwürdige, zwar „immer noch“ weitverbreitete, aber letztlich moralisch umstrittene Überzeugung, lässt sich mit den Methoden einer empirisch arbeitenden Biologie im Gegenteil leicht falsifizieren: Selbst unter der experimentellen Bedingung einer gendertheoriegeleiteten „sozialen Konstruktion von Dreigeschlechtlichkeit“ etwa bleibt Zweigeschlechtlichkeit weiter beobachtbar, wie vermutlich selbst ein einfacher Schülerversuch (etwa im Klassenzimmer) leicht und schlüssig beweisen könnte: Mädchen und Jungen blieben Jungen und Mädchen selbst unter der Bedingung, dass der Versuchsleiter sie z.B. in Mädchen, Jungen und Neutrale einordne. Hormonale Unterschiede während der Schwangerschaft, kurz nach der Geburt und während der Pubertät, ausgelöst durch genetische Determinanten, sind hierfür ursächlich (vgl. Meier, 2015). (Nicht nur) menschliche Zweigeschlechtlichkeit ist ein biologisches Faktum, sie lässt sich schlüssig theoretisch herleiten und empirisch nachweisen, die Grundannahme des Genderismus kann insofern als falsifiziert zurückgewiesen werden. Solch "wissenschaftliches" Insistieren auf gender kann kriminelle Züge annehmen, das zeigt der John/Joan-Fall D. Reimers und seines Arztes (ausführlich Meier 2015, S. 89; uneinsichtig Butler 2009). Mehr noch: Zweigeschlechtlichkeit hat im emotionalen Erleben und sozialen Handeln von Männern und Frauen kulturunabhängig bioevolutionär tiefste Spuren hinterlassen, sie ist kein soziales Konstrukt. Im Gegenteil, der wissenschaftsfremde Versuch einer „Dekonstruktion von Zweigeschlechtlichkeit“ wäre selbst als soziales Konstrukt zu dekonstruieren und auf seine versteckten und offenen (bildungs-)politischen 
Absichten hin zu prüfen. Das gälte insbesondere in Bezug auf seine faktische latente Männerfeindschaft und seine damit verbundenen sozialen Aufstiegsabsichten akademischer Frauen und ihrer akademischen und politischen Helfer und Helfershelfer, das gälte ebenso für die sich in ihr artikulierenden sexualpolitischen Partikularinteressen von Homosexuellen und Päderasten (vgl. Schmelcher, 2014). Dem widerspricht keineswegs, dass der Staat und seine Schulen sexuelle Vielfalt (etwa Hetero- und Homosexualität) als Gestaltungsbereich privater Lebensführung im Rahmen geltender Gesetze zu respektieren und zu achten haben, statt hier weitere Betätigungsfelder eines Paternalistischen Staates und Paternalistischer Schulen - unter dem Label einer empirisch fragwürdigen „sichtbaren oder vermuteten“ Diskriminierungsbeseitigung - seinerseits zu konstruieren.

Fachwissenschaftlich ist heute Konsens, dass die Zweigeschlechtlichkeit zum Zwecke des geneshuffling vor etwa 300 Millionen Jahren evolvierte. Phänomene wie Homosexualität, „guavedoces“, oder auch Transsexualität lassen sich biologisch bzw. medizinisch schlüssig mit der Zweigeschlechtlichkeitsthese vereinen (Campbell, 1972; Voland, 2009; Meier, 2015) - wird eine „politische Bewertung“ o. ä. gewünscht, so fällt sie nicht in den Zuständigkeitsbereich der Wissenschaft: Gesellschafspolitisch-philosophischer Spekulation sind auf dem Markt demokratisch vertretener Meinungen erfreulicherweise keine Grenzen zu setzen, in methodisch gelenkten Wissenschaften schon. Die These von der sozialen Konstruktion von Zweigeschlechtlichkeit, die genauso gut keine/unendliche Geschlechtlichkeiten umfasst oder umfassen könnte, ist empirisch absolut haltlos, sie gehört in den Bereich albern-pubertären Geschwätzes, ihre erstaunlichen Institutionalisierungsabsichten und -erfolge und ihre erschreckende Wirkungsmächtigkeit ändern daran nichts. Im Gegenteil: Es wird nicht reichen, auf einer fachwissenschaftlichen oder alltagslogischen Ebene kopfschüttelnd Genderismus zu ignorieren und darauf zu vertrauen, dass offensichtlicher Unfug sich schon von selbst erledigen wird.

Zusammenfassend kann konstatiert werden, dass einer politischen Indienstnahme von Bildungsinstitutionen entschieden widersprochen werden muss. Das historische Beispiel der Rassenkunde zeigte die verheerenden Auswirkungen, die aus einer Entwissenschaftlichung der Akademie resultierten, die sich in theoretisch unbegründeten Konzepten, einem Verzicht auf empirische Falsifizierbarkeit und den faktischen Rekurs auf politische Einflussnahme manifestierten. Gegenwärtig sind diese Gefahren durch kreationistische und genderistische Bestrebungen wieder virulent. Politisch erscheint der Genderismus die gefährlichere Variante dieser Bestrebungen zu sein. Allen diesen Versuchen sollte aktiv und couragiert Widerstand entgegengesetzt werden.

\section{Literatur}

Berger, Peter \& Luckmann, Thomas (1968/2003): Die gesellschaftliche Konstruktion der Wirklichkeit. Frankfurt/Main: Fischer.

Bundeszentrale für gesundheitliche Aufklärung (2015): Gender Mainstreaming im Kontext einer Sexualpädagogik der Vielfalt. In: http://forum.sexualaufklaerung.de/index.php?docid=667, Aufruf vom 25.9.2015.

Butler, Judith (1991): Das Unbehagen der Geschlechter. Frankfurt/Main: Suhrkamp.

Butler, Judith (2009): Die Macht der Geschlechternormen und die Grenzen des Menschlichen. Frankfurt/Main: Suhrkamp.

Campbell, Bernard Grant (1972): Sexual selection and the descent of man. 1871-1971. Ann Arbor (Mi.): Heinemann Educational.

Dawkins, Richard (2006): Der Gotteswahn. Berlin: Ullstein.

Dawkins, Richard (2010): The Greatest Show on Earth: The Evidence for Evolution. London: Free Press. 
DIE WELT vom 5.7.2007, zugänglich unter: http://www.welt.de/politik/article999798/ Schoepfungsgeschichte-im-Biologieunterricht.html, Aufruf vom 25.9.2015.

EKD-Pressemitteilung (2008): EKD zieht klare Trennlinie zum Kreationismus. In: http://www.ekd.de/aktuell_presse/news_2008_04_01_2_ekd_text_94.html, Aufruf vom 25.9.2015.

Günther, Hans Friedrich Karl (1935): Kleine Rassenkunde des deutschen Volkes. Bonn: Buchgemeinde. Hertz, Friedrich (1930): Hans Günther als Rassenforscher. Berlin: Philo.

Hacking, Ian (1999): Was heißt 'soziale Konstruktion'? Zur Konjunktur einer Kampfvokabel in den Wissenschaften. Frankfurt/Main: Fischer.

Jäger, Siegfried (2001): Diskurs und Wissen. Theoretische und Methodische Aspekte einer Kritischen Diskurs- und Dispositivanalyse In: Reiner Keller et al. (Hrsg.): Handbuch Sozialwissenschaftliche Diskursanalyse, Band I: Theorien und Methoden. Wiesbaden: Leske+Budrich, S. 81-112.

Kant, Immanuel (1781/1976): Kritik der reinen Vernunft. Frankfurt/Main: Suhrkamp.

Lauth, Bernhard \& Sareiter, Jamel (2002). Wissenschaftliche Erkenntnis. Eine ideengeschichtliche Einführung in die Wissenschaftstheorie. Paderborn: Mentis.

Meier, Markus (2015): Lernen und Geschlecht heute. Zur Logik der Geschlechterdichotomie in eduktativen Kontexten. Würzburg: Königshausen \& Neumann.

Ministerium für Schule und Weiterbildung des Landes Nordrhein-Westfalen (2015): Gendermainstreaming. In: http://www.schulministerium.nrw.de/docs/Schulsystem/Grundlagen/GenderMainstreaming/index.html, Aufruf vom 23.9.2015.

Popper, Karl (1935): Logik der Forschung. Wien: Springer.

Schmelcher, Antje (2014): Unter dem Deckmantel der Vielfalt. In: FAZ vom 14.X.2014.

Schwandt, Peter (2008): Hans F. K. Günther. Porträt, Entwicklung und Wirken des rassistischnordischen Denkens. Saarbrücken: VDM.

Voland, Eckart (20093): Soziobiologie: Die Evolution von Kooperation und Konkurrenz. Berlin: Spektrum.

Wegner, Gregory (1991): Schooling for a new mythos. Race, anti-semitism and the curriculum materials of a nazi race educator. In: Paedagogica historica, 27 (2), S. 189-213.

\section{Bilderverzeichnis}

Hans Friedrich Karl Günther, Umschlagblatt von Vererbung oder Erziehung, https://de.wikipedia.org/ wiki/Hans_F_K__G\%C3\%BCnther\#/media/File:G\%C3\%BCnther_Vererbung_od_Erziehung.jpg, Aufruf vom 25.9.2015

Stand einer „Anti-Evolutions-Liga“ in den Südstaaten der USA, 1925. http://www.nature.com/ polopoly_fs/7.3848.1334092721!/image/BE053411-630.jpg_gen/derivatives/landscape_300/ BE053411-630.jpg, Aufruf vom 25.9.2015

\section{Über den Autor}

Dr. Markus D. Meier: Professor für Allgemeine Erziehungswissenschaft an der Universidad Externado de Colombia in Bogotá (Kolumbien). Kontakt: markus.meier@uexternado.edu.co 